\title{
Avaliação da microbiota ocular em pacientes com disfunção do filme lacrimal
}

\section{Evaluation of conjunctival flora in patients with tear film dysfunction}

Melissa Megumi Tomimatsu', Manuela Maria Valença Cordeiro Barbosa², Cecília Zorat Yu³, Flavio Eduardo Hirai $^{4}$, Ana Luisa Höfling-Lima ${ }^{5}$

\begin{tabular}{l}
\hline$\underline{\text { RESUMO }}$ \\
Objetivo: Avaliar a microbiota conjuntival em olhos com disfunção do filme lacrimal, e \\
a modificação desta microbiota após a colocação de plug de silicone no canalículo \\
inferior. Métodos: Série de casos intervencionais não comparativos para avaliar 68 \\
olhos de 41 pacientes com disfunção do filme lacrimal, durante o período de 2002 a \\
2007, na Universidade Federal de São Paulo. Todos os pacientes foram submetidos à \\
colheita de amostras de raspado conjuntival de fundo-de-saco inferior para cultivo em \\
Brain heart infusion broth. Os vinte e dois pacientes submetidos à colocação de plug de \\
silicone repetiram a colheita de raspado conjuntival um mês após o procedimento. \\
Resultados: Dos 68 olhos avaliados, 47 apresentaram crescimento bacteriano nas amos- \\
tras colhidas. Nove diferentes espécies de bactérias foram identificadas: Staphylococcus \\
coagulase negativa em 66,66\%, Staphylococcus aureus em 13,72\%, Corynebacterium \\
sp em 5,86\%, Enterobacter aerogenes em 3,92\%, Streptococcus hemolítico do grupo \\
viridans em 1,96\%, Serratia sp em 1,96\%, Alcaligenes xylosoxidans spp em 1,96\%, \\
Corynebacterium xerosis em 1,96\%, e Proteus mirabilis em 1,96\%. Staphylococcus \\
coagulase negativa (SCN) foi o microrganismo mais frequentemente isolado tanto \\
antes quanto após o plug de silicone. A sensibilidade do SCN à Oxacilina antes da \\
colocação do plug era de 87,50\%, e, após, de 73,68\%.Conclusão: A microbiota em olhos \\
com disfunção do filme lacrimal é bastante semelhante à encontrada em olhos normais. \\
A resistência de SCN à Oxacilina foi um pouco maior após o implante do plug de \\
silicone.
\end{tabular}

Descritores: Síndrome do olho seco/microbiologia; Conjuntiva/microbiologia;

Infecções estafilocócicas /microbiologia; Elastômeros de silicone

\footnotetext{
${ }^{1}$ Médica Oftalmologista e Estagiária de Doenças Externas Oculares e Córnea do Departamento de Oftalmologia da Universidade Federal de São Paulo - UNIFESP - São Paulo (SP) - Brasil;

${ }^{2}$ Médica Oftalmologista e Estagiária de Doenças Externas Oculares e Córnea do Departamento de Oftalmologia da Universidade Federal de São Paulo - UNIFESP - São Paulo (SP) - Brasil;

${ }^{3}$ Biomédica responsável pelo Laboratório de Microbiologia do Departamento de Oftalmologia da Universidade Federal de São Paulo - UNIFESP - São Paulo (SP) - Brasil;

${ }^{4}$ Pós-graduando (doutorado) pela Universidade Federal de São Paulo - UNIFESP - São Paulo (SP) - Brasil;

${ }^{5}$ Livre-docente, Professora Titular do Departamento de Oftalmologia da Universidade Federal de São Paulo - UNIFESP - São Paulo (SP) - Brasil;

Departamento de Oftalmologia da Universidade Federal de São Paulo - UNIFESP - São Paulo (SP) - Brasil.
}

Recebido para publicação em: 7/7/2009 - Aceito para publicação em 5/11/2009 


\section{INTRODUÇÃO}

$\mathbf{0}$ filme lacrimal, além de lubrificar a superfície ocular, tem a importante função de protegê-la contra a ação de vários patógenos. A lágrima contém proteínas como a lisozima, a lactoferrina, imunoglobulina A, entre outras substâncias, que exercem atividade antimicrobiana ${ }^{(1-3)}$. Além disso, a mucina produzida por células caliciformes da conjuntiva promove uma barreira física que impede tanto a aderência como a penetração de muitos patógenos através do epitélio da superfície ocular ${ }^{(1-3)}$. Seal et al. mostraram que existe redução tanto da lactoferrina quanto da lisozima na lágrima de indivíduos com disfunção do filme lacrimal ${ }^{(4)}$.

Alguns estudos demonstraram um aumento da atividade da enzima metaloproteinase- 9 no epitélio corneano, em resposta à insuficiência do filme lacrimal, e que resultou em perda das "tight junctions" da barreira epitelial corneana ${ }^{(5)}$. A deficiência do filme lacrimal aliada à fragilidade epitelial corneana em olhos secos podem facilitar a instalação de infecções oculares.

Terapias anti-inflamatórias como esteróides tópicos e ciclosporina A têm sido prescritas em vários casos de disfunção do filme lacrimal, com melhora dos sintomas. A administração de imunomoduladores pode, contudo, alterar a atividade imune na superfície ocular, aumentando assim a predisposição à infeçcão ${ }^{(1)}$.

A oclusão de ponto lacrimal com plugs é uma opção terapêutica em casos de disfunção do filme lacrimal, uma vez que diminuem a drenagem lacrimal através dos canalículos ${ }^{(1,6,7)}$. Apesar de ser um método relativamente fácil e seguro, há estudos mostrando bactérias capazes de formar um biofilme aderente à superfície do plug $^{(6)}$.

O objetivo deste estudo foi avaliar a microbiota conjuntival em olhos com disfunção do filme lacrimal, e a modificação desta microbiota pela colocação do plug de silicone no canalículo inferior.

\section{Métodos}

Nesta série de casos intervencionais não comparativos foram avaliados 68 olhos de 41 pacientes ( 29 do sexo feminino, e 12 do sexo masculino) portadores de disfunção do filme lacrimal, durante o período de 2002 a 2007. Os critérios de exclusão foram pacientes em uso de antibioticoterapia ou de terapias anti-inflamatórias como corticóides ou imunossupressores tópicos ou sistêmicos. Foram incluídos pacientes com diagnóstico de disfunção do filme lacrimal em tratamento somente com lágrimas artificiais. $\mathrm{O}$ diagnóstico de disfunção do filme lacrimal foi feito pela presença de pelo menos um dos sintomas (sensação de olho seco, ardor ou dor ou sensação de corpo estranho, prurido e lacrimejamento), associados aos achados de exame oftalmológico (biomicroscopia avaliando-se conjuntiva e córnea, tempo de rompimento do filme lacrimal e teste com coloração vital rosa bengala).

$\mathrm{Na}$ biomicroscopia anterior foram valorizados sinais como presença de hiperemia conjuntival, muco, oleosidade excessiva do filme lacrimal, e de ceratite filamentar. Tempo de rompimento do filme lacrimal inferior a 10 segundos foi considerado indicativo de disfunção do filme lacrimal ${ }^{(8)}$. No teste com coloração vital rosa bengala foi instilada 1 gota da solução a $1 \%$ e o paciente foi orientado a piscar várias vezes. Para interpretação do resultado, sob luz anerita verde, a superfície ocular foi dividida em 3 partes: conjuntiva bulbar nasal e temporal e córnea. Cada área foi graduada de 0 a 3 pontos, em que "0" significava ausência de coloração e "3" correspondia à coloração confluente, para um máximo de 9 pontos em cada olho. Olhos com valores superiores a 3 pontos foram considerados portadores de disfunção do filme lacrimal ${ }^{(9)}$.

Durante o estudo, houve 22 pacientes ( 30 olhos), do total de 41 pacientes (68 olhos), que precisaram recorrer à oclusão de ponto lacrimal com plug de silicone. Estes pacientes foram submetidos à cultura de amostras colhidas do fundo-de-saco conjuntival antes e após um mês da oclusão do ponto lacrimal inferior.

\section{Colheita e Cultura}

Todos os pacientes tiveram amostras, para cultivo, obtidas do fundo-de-saco da conjuntiva inferior, com zaragatoa estéril; o material foi imediatamente inoculado em Brain heart infusion broth (BHI broth) (Difco, Le Pont de Claix/France) e encaminhado ao laboratório. Após apresentar turbidez, as amostras de BHI foram semeadas em meios sólidos de ágar sangue de carneiro a $5 \%$ (AS) (Difco, Le Pont de Claix/France) e ágar chocolate (AC) (Difco, Le Pont de Claix/France) para posterior identificação dos microrganismos presentes.

A sensibilidade aos antimicrobianos oxacilina, amicacina, tobramicina, ciprofloxacino, ofloxacino, gatifloxacino e moxifloxacino foi testada pelo método de disco difusão de acordo com as recomendações do Clinical Laboratory Standards Institute (CLSI, Filadélfia,EUA) e determinação das concentrações inibitórias mínimas por Etest ${ }^{\circledR}$ (AB Biodisk, Solna, Suécia).Todo o 
processamento das amostras e as respectivas análises microbiológicas foram realizados no laboratório de microbiologia ocular do departamento de Oftalmologia da Universidade Federal de São Paulo.

\section{Análise estatística}

Dados foram apresentados pela frequência, pelas médias \pm desvios-padrão ou proporções. Para comparação de variáveis categóricas foi utilizado o teste exato de Fisher. Valores de $p \leq 0,05$ foram considerados estatisticamente significantes. A análise foi realizada com o software Stata v.10 (College Park, Texas).

\section{Resultados}

A idade dos pacientes incluídos no estudo variou de 20 a 88 anos com média \pm desvio-padrão de $58,0 \pm$ 15,71 e mediana de 59 anos; $70,73 \%$ eram do sexo feminino e $29,27 \%$, do masculino.

Dos 68 olhos avaliados, 21 tiveram cultivos negativos e 47 apresentaram crescimento bacteriano nas amostras colhidas. Nove diferentes espécies de bactérias foram identificadas: Staphylococcus coagulase negativa $(\mathrm{SCN})$ em 34 amostras $(66,66 \%)$, Staphylococcus

Tabela 1

Microbiota dos 47 olhos com disfunção do filme lacrimal com cultura positiva

\begin{tabular}{lcc}
\hline Bactéria & Frequência & \% \\
\hline Staphylococcus coagulase negativa & 34 & 66,66 \\
Staphylococcus aureus & 7 & 13,72 \\
Corynebacterium sp & 3 & 5,86 \\
Enterobacter aerogenes & 2 & 3,92 \\
Corynebacterium xerosis & 1 & 1,96 \\
Streptococcus hemolítico do grupo viridans & 1 & 1,96 \\
Proteus mirabilis & 1 & 1,96 \\
Serratia sp & 1 & 1,96 \\
Alcaligenes xylosoxidans & 1 & 1,96 \\
Total & $51^{*}$ & 100 \\
\hline
\end{tabular}

* Dentre as 47 amostras, quatro tiveram crescimento de duas bactérias distintas

Tabela 2

Microbiota dos olhos com cultura positiva antes e após o implante de plug*

\begin{tabular}{lcccc}
\hline Bactéria & Pré-plug & \% & Pós-plug & \% \\
\hline Staphylococcus coagulase negativa & 16 & 64 & 19 & 70,38 \\
$\quad$ Sensível à oxacilina & $\mathbf{1 4}$ & $\mathbf{8 7 , 5 0}$ & $\mathbf{1 4}$ & $\mathbf{7 3 , 6 8}$ \\
Staphylococcus aureus & 4 & 16 & 1 & 3,70 \\
$\quad$ Sensível à oxacilina & $\mathbf{4}$ & $\mathbf{1 0 0}$ & $\mathbf{1}$ & $\mathbf{1 0 0}$ \\
Corynebacterium sp & 1 & 4 & 1 & 3,70 \\
Serratia sp & 1 & 4 & 1 & 3,70 \\
Streptococcus hemolítico do grupo viridans & 1 & 4 & 3 & 11,12 \\
Alcaligenes xylosidans spp & 1 & 4 & & \\
Corynebacterium xerosis & 1 & 4 & & \\
Citrobacter feundii & & & 1 & 3,70 \\
Moraxella nonliquefaciens & & & 1 & 3,70 \\
Total & 25 & 100 & 27 & 100 \\
\hline
\end{tabular}

*Alguns olhos apresentaram crescimento de mais de uma bactéria, conforme detalhado na Tabela 3 
Tabela 3

Microbiota antes e após o implante de plug nos 30 olhos dos 22 pacientes com disfunção do filme lacrimal submetidos ao procedimento

\begin{tabular}{|c|c|c|c|}
\hline ID & Olho & Microbiota pré-plug & Microbiota pós-plug \\
\hline \multirow[t]{5}{*}{1} & $\mathrm{D}$ & $\mathrm{SCN}$ & $\mathrm{SCN}$ \\
\hline & & & Citrobacter freundii \\
\hline & & & $\begin{array}{l}\text { Streptococcus hemolítico } \\
\text { do grupo viridans }\end{array}$ \\
\hline & $\mathrm{E}$ & $\mathrm{SCN}$ & SCN \\
\hline & & & $\begin{array}{l}\text { Streptococcus hemolítico } \\
\text { do grupo viridans }\end{array}$ \\
\hline 2 & $\mathrm{D}$ & $\mathrm{SCN}$ & SCN \\
\hline 3 & $\mathrm{D}$ & Corynebacterium spp & $\mathrm{SCN}$ \\
\hline 4 & $\mathrm{D}$ & $\mathrm{SCN}$ & $\mathrm{SCN}$ \\
\hline \multirow[t]{2}{*}{5} & $\mathrm{D}$ & $\mathrm{SCN}$ & $\mathrm{SCN}$ \\
\hline & $\mathrm{E}$ & $\mathrm{SCN}$ & $\mathrm{SCN}$ \\
\hline 6 & $\mathrm{E}$ & cultura negativa & cultura negativa \\
\hline \multirow[t]{2}{*}{7} & $\mathrm{D}$ & $\mathrm{SCN}$ & $\mathrm{SCN}$ \\
\hline & $\mathrm{E}$ & S. aureus & $\mathrm{SCN}$ \\
\hline 8 & $\mathrm{E}$ & S. aureus & $\mathrm{SCN}$ \\
\hline \multirow[t]{2}{*}{9} & $\mathrm{D}$ & SCN & cultura negativa \\
\hline & $\mathrm{E}$ & cultura negativa & cultura negativa \\
\hline 10 & $\mathrm{D}$ & Alcaligenes xylosoxidans spp & $\mathrm{SCN}$ \\
\hline 11 & $\mathrm{E}$ & cultura negativa & Corynebacterium spp \\
\hline 12 & $\mathrm{D}$ & $\mathrm{SCN}$ & $\mathrm{SCN}$ \\
\hline \multirow[t]{2}{*}{13} & $\mathrm{D}$ & Serratia spp & cultura negativa \\
\hline & $\mathrm{E}$ & cultura negativa & Serratia spp \\
\hline \multirow[t]{2}{*}{14} & $\mathrm{E}$ & $\mathrm{SCN}$ & Streptococcus hemolítico \\
\hline & & $\begin{array}{l}\text { Streptococcus hemolítico } \\
\text { do grupo viridans }\end{array}$ & do grupo viridans \\
\hline 15 & $\mathrm{E}$ & Corynebacterium xerosis & cultura negativa \\
\hline 16 & $\mathrm{E}$ & cultura negativa & $\mathrm{SCN}$ \\
\hline \multirow[t]{3}{*}{17} & $\mathrm{D}$ & $\mathrm{SCN}$ & $\mathrm{SCN}$ \\
\hline & & & S. aureus \\
\hline & $\mathrm{E}$ & $\mathrm{SCN}$ & $\mathrm{SCN}$ \\
\hline 18 & $\mathrm{D}$ & cultura negativa & cultura negativa \\
\hline 19 & $\mathrm{E}$ & SCN & $\mathrm{SCN}$ \\
\hline \multirow[t]{2}{*}{20} & $\mathrm{D}$ & S. aureus & cultura negativa \\
\hline & $\mathrm{E}$ & S. aureus & cultura negativa \\
\hline \multirow[t]{2}{*}{21} & $\mathrm{E}$ & SCN & $\mathrm{SCN}$ \\
\hline & & & Moraxella nonliquefaciens \\
\hline \multirow[t]{2}{*}{22} & $\mathrm{D}$ & $\mathrm{SCN}$ & SCN \\
\hline & $\mathrm{E}$ & $\mathrm{SCN}$ & $\mathrm{SCN}$ \\
\hline
\end{tabular}

aureus em 7 (13,72\%), Corynebacterium sp em 3 $(5,86 \%)$, Enterobacter aerogenes em $2(3,92 \%)$, Streptococcus hemolítico do grupo viridans em 1 (1,96\%), Serratia sp em 1 (1,96\%), Alcaligenes xylosoxidans spp em $1(1,96 \%)$, Corynebacterium xerosis em $1(1,96 \%)$, e Proteus mirabilis em 1 (1,96\%) (Tabela 1). Dos 47 olhos que apresentaram cultivo positivo, isolou-se uma única espécie de bactéria em 43 amostras, e em 4, duas espécies de bactérias foram isoladas.

A oclusão de ponto lacrimal com plug de silicone foi efetuada em 30 olhos de 22 pacientes (77,3\% do sexo feminino e $22,7 \%$ do sexo masculino).
Dos 30 olhos, 24 (80\%) tiveram cultura positiva antes da colocação do plug, sendo $64 \%$ para SCN, $16 \%$ para S. aureus, e $20 \%$ para outras bactérias (Streptococcus hemolítico do grupo viridans, Corynebacterium sp, Serratia sp, Alcaligenes xylosoxidans spp) (Tabela 2).

Após o implante do plug, 22 (73,3\%) dos 30 olhos apresentaram crescimento bacteriano, sendo que, em quatro deles, houve crescimento de mais de uma bactéria (Tabela 3). SCN continuou sendo o microrganismo mais prevalente, com $70,38 \%$, seguido pelo Streptococcus hemolítico do grupo viridans, com $11,12 \%$, e pelos outros (S. aureus, Serratia sp, Alcaligenes 
Tabela 4

Perfil de sensibilidade aos antimicrobianos das bactérias isoladas de pacientes com disfunção do filme lacrimal

\begin{tabular}{lcccc}
\hline & \multicolumn{2}{c}{$\begin{array}{c}\text { SCN } \\
(\mathbf{N = 3 4 )}\end{array}$} & \multicolumn{2}{c}{$\begin{array}{c}\text { S. aureus } \\
(\mathbf{N = 7 )}\end{array}$} \\
\cline { 2 - 5 } & $\mathbf{n}$ & $\mathbf{0}$ & $\mathbf{n}$ & \% \\
\hline Oxacilina & 30 & 88,23 & 7 & 100 \\
Amicacina & 33 & 97,05 & 7 & 100 \\
Tobramicina & 28 & 82,35 & 7 & 100 \\
Ciprofloxacino & 29 & 85,29 & 7 & 100 \\
Ofloxacino & 32 & 94,11 & 7 & 100 \\
Gatifloxacino & 29 & 85,29 & 6 & 85,71 \\
Moxifloxacino & 29 & 85,29 & 6 & 85,71 \\
\hline
\end{tabular}

$\mathrm{N}=$ número de isolados; $\mathrm{n}=$ número de isolados sensíveis

xylosidans spp, Corynebacterium sp, Moraxella nonliquefaciens, e Citrobacter feundii) com 18,5\% (Tabela 2).

Houve mudança na microbiota de 17 olhos $(56,6 \%)$ após o implante do plug, mas isto não teve associação com alterações de sinais e sintomas.

Observou-se, neste estudo, que nos olhos com disfunção do filme lacrimal, 88,23\% dos SCN eram sensíveis à oxacilina, $82,35 \%$ eram sensíveis à tobramicina e $85,29 \%$ à ciprofloxacino ou a moxifloxacino (Tabela 4).

Nos olhos que necessitaram de plug, $87,5 \%$ dos SCN eram sensíveis à oxacilina antes, e 73,68\% após a colocação do plug de silicone.

\section{$\underline{\text { DiscusSÃo }}$}

O presente estudo confirma dados anteriormente publicados sobre a microbiota de pacientes com disfunção do filme lacrimal, que apresentam maior prevalência de Staphylococcus coagulase negativa (SCN) com ou sem oclusão do ponto lacrimal com plug de silicone ${ }^{(1,10)}$. Diversos autores também demonstraram que SCN é o microrganismo mais prevalente em olhos normais ${ }^{(11,12)}$.Ainda não há estudos descrevendo alterações da microbiota de acordo com a severidade da disfunção lacrimal.

O método utilizado para avaliar a microbiota, neste estudo, foi o de cultivo em meios sólidos e líquidos. Estudos com PCR descrevem que este método é capaz de detectar uma diversidade muito maior de bactérias tanto na superfície de olhos saudáveis quanto na de olhos com disfunção do filme lacrimal, enquanto que os métodos de cultivo convencionais frequentemente apresentam resultados negativos ou somente positivos para
$\mathrm{SCN}^{(10)}$. Isso ocorre porque o $\mathrm{PCR}$ pode detectar material genético mesmo de microrganismos com difícil crescimento em meios de cultura convencionais, especialmente devido à limitada quantidade do microrganismo na amostra. Entretanto, a elevada sensibilidade do exame molecular permite a detecção de microrganismos que, apesar de presentes, podem não estar relacionados ao quadro clínico. É muito difícil instituir um tratamento na ausência de sinais e sintomas oculares.

É frequente que o paciente com disfunção do filme lacrimal apresente-se com sinais de olho vermelho e sintomas de irritação ocular ${ }^{(5,13)}$. As enzimas e toxinas produzidas por muitas das bactérias que colonizam a superfície ocular são capazes de destruir a camada de mucina e de lipídios, desestabilizando ainda mais o filme lacrimal, e agravando a inflamação ocular e os sintomas de olho com disfunção do filme lacrimal ${ }^{(3,10)}$. Em olhos com inflamação crônica há maior risco de existirem SCN multirresistentes ${ }^{(1,14)}$. Em contrapartida, neste estudo, observou-se que, em olhos com disfunção do filme lacrimal, 87,50\% dos $\mathrm{SCN}$ eram sensíveis à oxacilina. Após a oclusão do ponto lacrimal inferior com plug de silicone, ainda $73,68 \%$ dos $\mathrm{SCN}$ isolados eram sensíveis à oxacilina.

Neste estudo, apesar de 17 (56,6\%) dos 30 olhos dos 22 pacientes submetidos ao plug terem apresentado alteração da microbiota, não foi observado um padrão de modificação no perfil de microrganismos encontrados em olhos com disfunção do filme lacrimal após o implante do plug de silicone. Além disso, não é possível interpretar se o plug foi o fator determinante da mudança. Para tanto, o ideal seria que cada paciente submetido ao implante de plug tivesse um olho com plug e o outro sem, como controle.

Sabe-se que a possibilidade de formação de 
biofilme é frequente em portadores de plug de silicone. O biofilme pode resultar em maior resistência a antibióticos ${ }^{(6,15)}$. Este fator de formação de biofilme não foi avaliado neste estudo. Entretanto, houve o cuidado de não se tocar o plug durante a colheita das amostras.

A disfunção do filme lacrimal geralmente ocorre em pacientes com idade mais avançada, que eventualmente precisa ser submetida a cirurgias oculares. A presença de bactérias sensíveis antes e depois da colocação do plug - identificadas neste estudo aparentemente não ressalta a necessidade de mudança de conduta terapêutica no pré-operatório desses pacientes.

\section{Conclusão}

Há poucos estudos sobre a microbiota em olhos com disfunção do filme lacrimal com ou sem plug de silicone, especialmente em nossa população.

Este estudo detectou maior prevalência de SCN na microbiota conjuntival de pacientes de nosso serviço com disfunção do filme lacrimal. Os pacientes submetidos ao implante de plug de silicone, apesar da modificação da microbiota, não evoluíram clinicamente com infecção de superfície ocular.

\section{Abstract}

Purpose: To evaluate conjunctival microbiota in eyes with tear film dysfunction and its modification after punctal occlusion with silicone plug. Methods: Non comparative interventional case series study to evaluate 68 eyes of 41 patients with tear film dysfunction, from 2002 to 2007, followed in Federal University of Sao Paulo. Samples for culture were all obtained from conjunctival swabs and inoculated in Brain heart infusion broth (BHI broth). Twenty two patients that undergone punctal plug occlusion repeated culture procedure one month after plug insertion.

Results: 47 of the 68 eyes evaluated had positive culture in their samples. Nine different types of bacteria were identified: Coagulase negative Staphylococcus in 66,66\%, Staphylococcus aureus, in 13,72\%, Corynebacterium sp, in 5,86\%, Enterobacter aerogenes, in 3,92\%, Streptococcus hemolitico do grupo viridans, in 1,96\%, Serratia sp, in 1,96\%, Alcaligenes xylosoxidans spp, in 1,96\%, Corynebacterium xerosis, in 1,96\%, and Proteus mirabilis in 1,96\%. Coagulase negative Staphylococcus (CNS) was the most frequently isolated microorganism before and after punctal occlusion. CNS sensibility to
Oxacilin before plug insertion was $87,50 \%$, and after, $73,68 \%$. Conclusion: Microbiota found in eyes with tear film dysfunction seems to be similar to that found in normal eyes. CNS resistance to oxacilin was slightly higher after silicone plug insertion.

Keywords: Dry eye syndromes/microbiology; Conjunctiva/microbiology; Staphylococcal infections/ microbiology; Silicone elastomers

\section{RefERÊNCIAS}

1. Hori Y, Maeda N, Sakamoto M, Koh S, Inoue T, Tano Y. Bacteriologic profile of the conjunctiva in the patients with dry eye. Am J Ophthalmol. 2008;146(5):729-34.

2. Lemp MA. understanding and managing dry eye disease. Am J Ophthalmol. 2008;146(3):350-6.

3. Berry M, Harris A, Lumb R, Powell K. Commensal ocular bacteria degrade mucins. Br J Ophthalmol. 2002;86(12):1412-6.

4. Seal DV, McGill JI, Mackie IA, Liakos GM, Jacobs P, Goulding NJ. Bacteriology and tear protein profiles of the dry eye. $\mathrm{Br}$ J Ophthalmol. 1986 70(2):122-5.

5. Pflugfelder SC, Farley W, Luo L, Chen LZ, de Paiva CS, Olmos LC, et al. Matrix metalloproteinase-9 knockout confers resistance to corneal epithelial barrier disruption in experimental dry eye. Am J Pathol. 2005;166(1):61-71.

6. Sugita J, Yokoi N, Fullwood NJ, Quantock AJ, Takada Y, Nakamura Y, et al. The detection of bacteria and bacterial biofilms in punctal plug holes. Cornea. 2001;20(4):362-5.

7. Horwath-Winter J, Thaci A, Gruber A, Boldin I. Long-term retention rates and complications of silicone punctal plugs in dry eye. Am J Ophthalmol. 2007;144(3):441-4.

8. N RENPK-J. Olho Seco ou Ceratoconjuntivite Sicca. In: N. K-J, editor. Doenças da córnea e conjuntiva. Rio de Janeiro: Cultura Médica; 2007. p. 275-84.

9. van Bijsterveld OP. Diagnostic tests in the Sicca syndrome. Arch Ophthalmol. 1969;82(1):10-4.

10. Graham JE, Moore JE, Jiru X, Moore JE, Goodall EA, Dooley JS, et al. Ocular pathogen or commensal: a PCR-based study of surface bacterial flora in normal and dry eyes. Invest Ophthalmol Vis Sci. 2007 Dec;48(12):5616-23.

11. Moeller CT, Branco BC, Yu MC, Farah ME, Santos MA, Hofling-Lima AL. Evaluation of normal ocular bacterial flora with two different culture media. Can J Ophthalmol. 2005 Aug;40(4):448-53.

12. Mino de Kaspar H, Koss MJ, He L, Blumenkranz MS, Ta CN. Antibiotic susceptibility of preoperative normal conjunctival bacteria. Am J Ophthalmol. 2005 Apr;139(4):730-3.

13. Solomon A, Dursun D, Liu Z, Xie Y, Macri A, Pflugfelder SC. Pro- and anti-inflammatory forms of interleukin-1 in the tear fluid and conjunctiva of patients with dry-eye disease. Invest Ophthalmol Vis Sci. 2001 Sep;42(10):2283-92.

14. Grasbon T, Mino de Kaspar H, Klauss V. [Coagulase-negative staphylococci in normal and chronically inflamed conjunctiva]. Ophthalmologe. 1995 Dec;92(6):793-801.

15. John JF, Harvin AM. History and evolution of antibiotic resistance in coagulase-negative staphylococci: Susceptibility profiles of new anti-staphylococcal agents. Ther Clin Risk Manag. 2007 Dec;3(6):1143-52. 